\title{
Síndrome asmático crítico en Pediatría: manejo de las exacerbaciones agudas severas Artículo de revisión
}

\author{
Ramírez-Esquivel David Ubaldo*
}

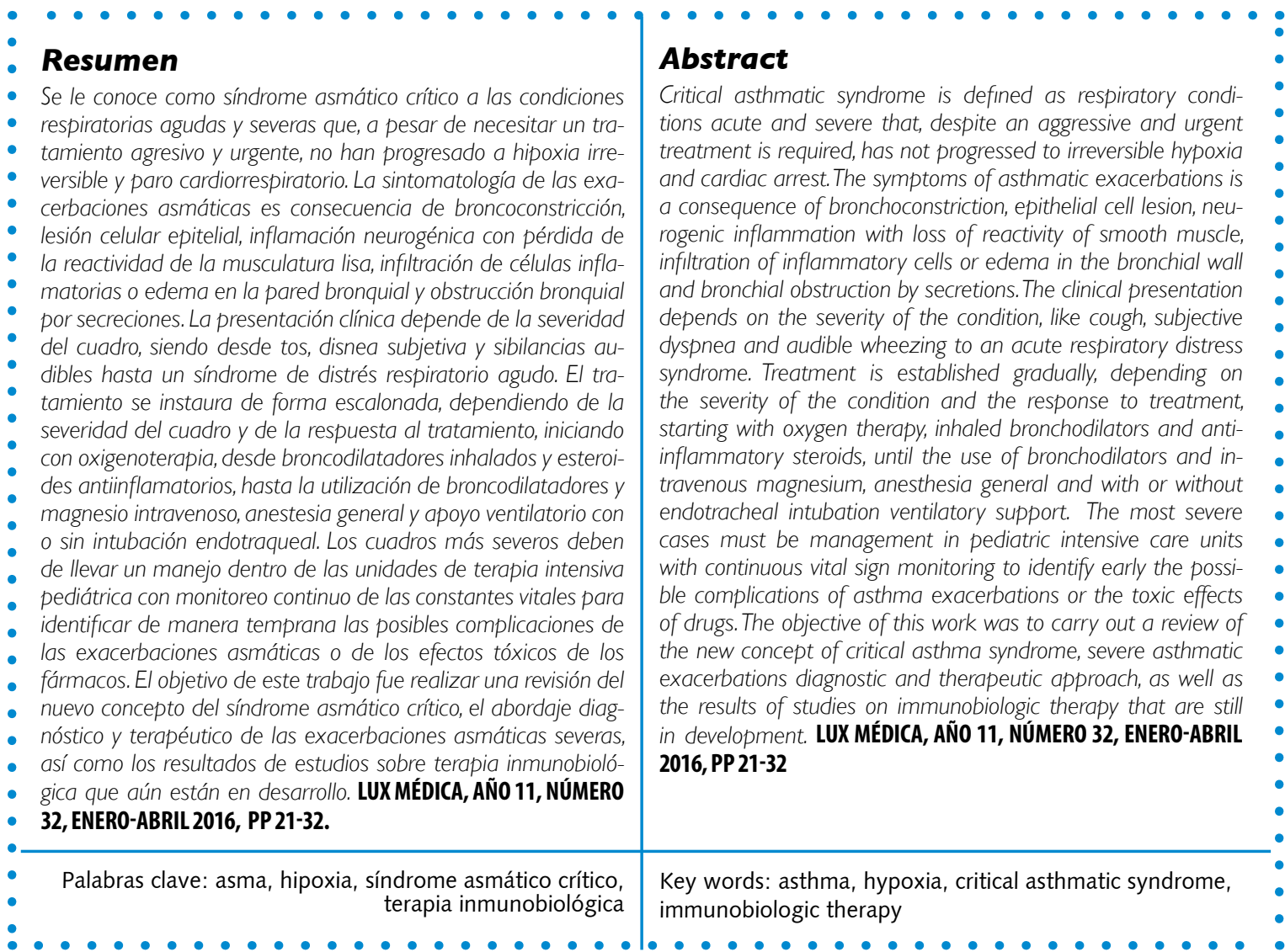

Pasante de Servicio Social en Investigación de la carrera de Médico Cirujano de la Universidad Autónoma de Aguascalientes.

Fecha de recibido: 9 de diciembre 2015

Fecha de aceptación: 9 de febrero 2016

Correspondencia: David Ubaldo Ramírez Esquivel. Laboratorio de Neuroinmunoendocrinología Departamento de Fisiología y Farmacología. Centro de Ciencias Básicas. Universidad Autónoma de Aguascalientes. Avenida Universidad \# 940. Colonia Ciudad Universitaria. Aguascalientes, Aguascalientes. México. Código Postal 20131. Correo electrónico davidre2000@hotmail.com 


\section{Introducción}

El asma es una enfermedad heterogénea que generalmente se caracteriza por una inflamación crónica de las vías aéreas ${ }^{1}$. Se define por las manifestaciones clínicas de síntomas respiratorios como sibilancias, dificultad respiratoria, opresión torácica y tos, que varían a lo largo del tiempo y en su intensidad, junto con una limitación variable del flujo aéreo ${ }^{1,2}$

Recientemente, se ha utilizado el término síndrome asmático crítico en las condiciones respiratorias agudas y severas que, a pesar de necesitar un tratamiento agresivo y urgente, no han progresado a hipoxia irreversible y paro cardiorrespiratorio. Este síndrome incluye descompensaciones asmáticas agudas y subagudas caracterizadas por signos y síntomas inespecíficos (asma aguda severa, asma refractaria, estado asmático, asma casi fatal) como disnea, fatiga respiratoria progresiva y ansiedad ${ }^{3}$.

La prevalencia del asma varía de acuerdo con la zona geográfica, el clima, el estilo de vida y el desarrollo económico de cada región ${ }^{4}$. Se le considera como la enfermedad crónica más común de la infancia. La prevalencia en América Latina se ha calculado en $5.7 \%$ a $16.5 \%$ mientras que en los estudios realizados en los últimos años en la Ciudad México aplicando la metodología del ISAAC (International Study of Asthma and Allergies in Childhood) revelan una prevalencia que va desde $8 \%$ a $12 \%{ }^{5}$. El asma es una de las causas de hospitalización más frecuente en pediatría. En el año 2000, se registraron alrededor de 152 mil hospitalizaciones por asma en un hospital de Estados Unidos, generando un costo total de 835 millones de dólares ${ }^{6}$. Si bien las exacerbaciones asmáticas severas son un motivo común para el ingreso a una unidad de cuidados intensivos $(\mathrm{UCl})$, sólo el $8 \%$ de los pacientes pediátricos con asma requerirán la admisión en una unidad de cuidados intensivos pediátricos (UCIP) ${ }^{7}$.

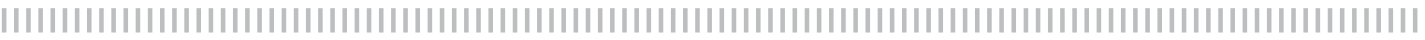

\section{Fisiopatología}

Después de la exposición a un desencadenante de respuesta asmática inmediata inicia la degranulación de los mastocitos y la activación de linfocitos $\mathrm{T}$ subtipo $\mathrm{TH} 2$, estos a su vez amplían el proceso inflamatorio a través de la producción de citocinas y quimiocinas: IL-4, IL-5, IL-8, IL-13. Las IL-4 e IL-13 favorecen la producción y liberación de IgE por parte de las células plasmáticas. Esta inmunoglobulina favorece la liberación de leucotrienos e IL-5 por parte de los mastocitos, promoviendo así la ma- duración y degranulación de los eosinófilos activados en la vía aérea ${ }^{8-10}$

Al seguir el proceso inflamatorio existe sobreproducción de moco y destrucción epitelial que desnuda la superficie de la vía aérea, exponiendo las terminaciones nerviosas. Esto induce hiperreactivdad bronquial y hace que la vía aérea sea más susceptible al broncoespasmo y a la obstrucción mediante la exposición a factores desencadenantes, convirtiéndose en un circuito cerrado que culmina en la remodelación bronquial permanente por el proce- 
so inflamatorio crónico (engrosamiento de la membrana basal reticular por depósito de colágeno, hiperplasia de las células caliciformes y la hipertrofia e hiperplasia del músculo liso bronquial) con aumento del espesor de las paredes de las vías respiratorias, disminución del calibre y distensibilidad, y un aumento en las resistencias al flujo aéreo. Estos fenómenos causan una obstrucción permanente de las vías respiratorias, con aumento de la respuesta bronquial y reducción acelerada de la función pulmonar ${ }^{8-13}$.

La obstrucción de la vía aérea se debe a la contribución de diferentes factores, cada uno en mayor o menor medida: 1) broncoconstricción; 2) lesión celular epitelial; 3) inflamación neurogénica con pérdida de la reactividad de la musculatura lisa; 4) infiltración de células inflamatorias o edema en la pared bronquial; y 5) obstrucción luminal por secreciones ${ }^{12,13}$. Estos factores perturban la mecánica pulmonar, incrementan los volúmenes pulmonares, provocan un desbalance ventilación/perfusión e incrementan los requerimientos de oxígeno.

La oclusión de las vías aéreas en áreas extensas de unidades alveolares, donde la ventilación es reducida severamente pero la perfusión es mantenida, incrementa los cortocircuitos o puede producir un incremento del espacio muerto pulmonar o hiperinflación alveolar regional cuando se asocia a una perfusión reducida. Estos cambios se traducen en un incremento de la frecuencia respiratoria y un volumen minuto elevado ${ }^{12}$.

En casos más severos, existe prolongación del tiempo inspiratorio e inicio de la inspiración antes de que la exhalación finalice, lo que provoca atrapamiento aéreo con hiperinflación alveolar. La espiración se convierte en proceso activo por el uso de los músculos accesorios de la respiración por la resistencia alta de flujo de aire $^{12,13}$. La presión negativa intrapleural incrementada durante la inspiración favo- rece el movimiento del líquido de edema capilar a los espacios aéreos ${ }^{11,13}$. Esto causa una fase de incremento en la poscarga ventricular derecha y una disminución de la precarga ventricular izquierda ${ }^{13}$, con un descenso en el gasto cardiaco clínicamente manifestado por el pulso paradójico ${ }^{12,13}$. La poscarga ventricular derecha se ve incrementada como resultado de la hipoxia severa y la acidosis; un estado de resistencia vascular pulmonar aumentado secundario a una hiperinflación dinámica ${ }^{12,13}$.

Aunque la fisiopatología del asma aguda en niños y adultos es similar, los niños son especialmente susceptibles a padecer exacerbaciones agudas severas ${ }^{14}$ : 1) los niños menores de 5 años poseen una vía aérea con mayor resistencia que la de los adultos; 2) un pequeño estrechamiento de las vías aéreas periféricas provoca aumentos desproporcionados en la resistencia al flujo del aire; 3) su reserva respiratoria es limitada y aumenta con la edad por aumento de tamaño de las vías respiratorias; 4) poseen un área de superficie respiratoria menor; 5) sus vías respiratorias poseen menor elasticidad con mayor propensión al colapso; 6) el reclutamiento diafragmático es menos eficiente por su inserción horizontal.

Las infecciones virales del tracto respiratorio superior son la principal causa de las exacerbaciones del asma ${ }^{15}$, constituyendo éstas del $85 \%$ al $95 \%$ de las exacerbaciones agudas en niños ${ }^{16}$. De las infecciones virales que con mayor frecuencia se asocian a exacerbaciones del asma se encuentran las infecciones por rinovirus ${ }^{17}$, que se ha encontrado en el $29 \%$ de las exacerbaciones del asma, de los cuales el $47 \%$ se hospitalizan con cuadros que entran en el concepto de un síndrome asmático crítico $^{16}$. El virus sincitial respiratorio sólo se ha asociado a exacerbaciones del asma en un $3 \%{ }^{18}$.

Recientemente se ha encontrado la presencia de bacterias atípicas, Mycoplasma 
pneumoniae y Chlamydia pneumoniae, en las vías respiratorias de personas asmáticas, proponiendo que la presencia persistente de estos organismos dentro de las vías aéreas pudiera contribuir en el desarrollo del asma en personas susceptibles e influyendo en el fenotipo clínico ${ }^{19}$.

Además de las infecciones respiratorias, existen otros factores de riesgo que aumentan la probabilidad de sufrir una crisis asmática, como lo son: pobre control de la sintoma- tología crónica, historia de una exacerbación severa en el último año que requirió intubación o ingreso a la Unidad de Cuidados Intensivos Pediátricos (UCIP), exposición al humo de tabaco, un volumen espiratorio forzado en el primer segundo (FEV1) menor de $60 \%$, problemas psicológicos (ansiedad generalizada, crisis de pánico) o sociales (bajo nivel socioeconómico), alergia alimentaria y/o de otro tipo confirmadas, exposición a otros alergenos y eosinofilia en expectoración ${ }^{1}$.

\section{| | | | | | | | | | | | | | | | | | | | | | | | | | | | | | | | | | | | | | | | | | | | | | | | | | | | | | | | | | | | | | | | | | | | | | | | | | | | | | | | | | | | | | | | | | | | | | | | | | | | | | | | | | || ||}

\section{Diagnóstico}

La evaluación inicial en la sala de urgencias se deberá realizar bajo el concepto universal $A B C$ (vía aérea, respiración y circulación; del inglés Airway, Breathing, Circulation), aplicándolo principalmente a pacientes con asma sintomática severa ${ }^{20}$. Es importante investigar la exposición a un posible factor desencadenante, el uso de medicamentos en casa (o la falta de los mismos), lo cual ayudará a determinar el cumplimiento y control de la enfermedad de base; investigar la "regla de los dos" (uso de medicamentos de rescate más de dos veces por semana, presencia de síntomas nocturnos más de dos veces al mes, recargar los medicamentos de rescate más de dos veces al año), que nos ayudará a evaluar la gravedad y el control de la enfermedad, así como también el antecedente de crisis asmática severa que requirió de intubación y ventilación mecánica, el número de visitas a urgencias en el año previo, el curso más reciente de esteroides sistémicos, el número total de ciclos de esteroides en el último año y presencia de alergias alimentarias ${ }^{23}$. La presentación de las exacerbaciones agudas varía según la gravedad, el factor desencadenante y la edad del paciente. Los síntomas más frecuentes en los niños son tos, sibilancias, disnea y ansiedad. Puede presentarse diaforesis en reposo, incapacidad para permanecer en decúbito supino y hablar con oraciones o frases cortas. En casos graves puede haber cianosis evidente de tipo central, embotamiento y disfunción cardiovascular. La somnolencia y confusión son signos de parada respiratoria inminente ${ }^{21}$, así como la disminución de las sibilancias y sonidos respiratorios con un "silencio torácico" en un paciente con aumento del esfuerzo respiratorio 22

Los signos que se pueden encontrar durante la exploración física son cianosis central, uso de músculos respiratorios accesorios y sibilancias audibles 0 , en caso de que la vía aérea esté demasiado obstruida, espiración prolongada ${ }^{21}$. Las retracciones manifestadas por el uso de los músculos accesorios de la respiración (en espacial las retracciones supraclaviculares) indican una predicción de una FEV $1<50 \%$ y debe alertar al médico de una enfermedad grave $^{23}$. Se puede encontrar en la auscultación sonidos espiratorios de alta y baja frecuencia ${ }^{22}$. Los signos que orientan hacia una obstrucción de las vías respiratorias significativa y/o fatiga de los músculos de la respiración son taquicardia y pulso paradójico ${ }^{21}$. La presencia de pulso paradójico se correlaciona con la gravedad de la crisis asmática y puede ser útil en el control de la gravedad de la enfermedad ${ }^{24}$.

Las radiografías de tórax se deben de tomar a aquellos niños con sibilancias de primera vez, con evidencia clínica de enfer- 
medad del parénquima pulmonar o aquellos que requieren ingreso a la unidad de cuidados intensivos pediátricos ${ }^{24}$, además aquellos que presenten fiebre $>39^{\circ} \mathrm{C}$, hipoxia severa, alteraciones focales durante la exploración física, sin antecedentes familiares de asma o aquellos que responden de forma inadecuada a la terapia broncodilatadora $^{23}$. La radiografía de tórax en el síndrome asmático crítico se puede usar en búsqueda de complicaciones subyacentes, como neumonía o fugas de aire ${ }^{22}$, o en casos de que la causa subyacente de las sibilancias esté en duda ${ }^{24}$, como la sospecha de cuerpo extraño en la vía aérea.

La oximetría de pulso es una medida fiable y no invasiva de la oxigenación y se debe utilizar en todos los pacientes para guiar la administración de suplementos de oxígeno. Sin embargo, la saturación de oxígeno no es un buen parámetro de una ventilación adecuada en los niños que reciben tratamiento con oxígeno ${ }^{22}$.

Los análisis de gases en sangre arterial deben de ser de rutina en pacientes ingresados a una unidad de cuidados intensivos. En etapas tempranas de los ataques graves, la hipoxia es un hallazgo universal $\left(\mathrm{PaO}_{2}<60 \mathrm{mmHg}\right.$, saturación de oxígeno $<95 \%)^{21}$, y en algunos casos puede encontrarse alcalosis respiratoria con hipocapnia causada por hiperventilación compensatoria $y$, mientras la enfermedad progresa, puede presentarse una acidosis metabóli$\mathrm{ca}$, ya sea sola o como parte de una acidosis mixta ${ }^{21}$, debido a la fatiga muscular y/o a la incapacidad de mantener una adecuada ventilación alveolar y la producción de lactato por el intenso trabajo muscular. En pacientes en los que se presenta una transición de hipercapnia a normocapnia pueden ser indicadores importantes de deterioro clínico ${ }^{22}$, debidos a obstrucción severa y pueden indicar la necesidad de ventilación mecánica ${ }^{21}$. Una $\mathrm{PaO}_{2}<60 \mathrm{mmHg}$ y una $\mathrm{PaCO}_{2}$ normal o aumentada ( $>45$ $\mathrm{mmHg}$ ) indica la presencia de insuficiencia respiratoria ${ }^{24}$. Los niños que se encuentran intubados requerirán frecuentes mediciones de gases arteriales con el fin de seguir la evolución clínica ${ }^{21}$.

Se debe de medir la concentración sérica de electrolitos y de glucosa, ya que hasta el $70 \%$ de los pacientes pueden cursar con hipocalemia secundaria a las modificaciones del $\mathrm{pH}$ sérico $\mathrm{y} / \mathrm{o}$ al uso de beta agonistas y corticoesteroides; hiponatremia por secreción inapropiada de la hormona antidiurética secundaria a la liberación de vasopresina por hipoxemia e hipercapnia, además de que el uso de esteroides pueden causar hiponatremia por efecto mineralocorticoide; y modificaciones en el metabolismo de los carbohidratos por el efecto de medicamentos usados en el tratamiento de las crisis asmáticas (beta agonistas y corticoesteroides) ${ }^{12}$.

La determinación de la cuenta leucocitaria y desviaciones hacia la izquierda pudiesen indicar una infección bacteriana como posible desencadenante de la crisis asmática y la posibilidad del uso de antibióticos como parte del tratamiento de la crisis, pero se debe de tener en cuenta que una leucocitosis puede estar relacionada con un fenómeno de estrés adaptativo o como efecto secundario del uso de corticoesteroides $^{12}$.

Las pruebas de función pulmonar ayudan a evaluar el grado de obstrucción al flujo aéreo. El volumen espiratorio forzado en un segundo (FEV1), la capacidad vital forzada (FVC) y la relación FEV1/FVC se utilizan para evaluar la gravedad del asma. La tasa de flujo espiratorio máximo (FEM) de menos de $50 \%$ del valor predicho indica una obstrucción grave del flujo aéreo, y menos de $33 \%$ del valor predicho sugiere un alto riesgo para el desarrollo de la hipercapnia y la hipoxemia ${ }^{21}$. 


\section{Tratamiento}

Los objetivos del tratamiento son: 1) corrección de la hipoxemia mediante la administración de $\mathrm{O}_{2} ; 2$ ) alivio de la obstrucción de la vía aérea, a través de la administración repetida de broncodilatadores inhalados; y 3) la disminución de la inflamación, así como la prevención de las recaídas mediante la administración de corticoides sistémi$\cos ^{25}$ (figura 1).

Los pacientes con exacerbaciones asmáticas severas deben de ser admitidos en una unidad de cuidados intensivos pediátricos para una monitorización y tratamiento ópti$\operatorname{mos}^{21}$. Se monitorizarán los signos vitales de forma continua para la identificación oportuna de complicaciones de las crisis asmáticas o de efectos secundarios del tratamiento de las mismas que puedan comprometer la adecuada evolución de los pacientes ${ }^{26}$.

Se debe de corregir la hipoxemia usando suplemento de oxígeno por cánula nasal o con tienda facial, acompañado de ambiente húmedo y alto flujo de oxígeno ${ }^{21}$. Se sugiere iniciar la oxigenoterapia con la fracción inhalada de oxígeno $\left(\mathrm{FIO}_{2}\right)$ del $24 \%$ con un flujo constante de 1 litro por minuto'. Se debe mantener hasta obtener cifras de saturación de oxígeno alrededor de 94\%$98 \%{ }^{1,27}$. Algunos autores consideran el oxígeno como un fármaco en el asma aguda, ya que disminuye la vasoconstricción pulmonar hipóxica e interfiere con la relación ventilación/perfusión características de una broncoconstricción severa ${ }^{22}$.

Las exacerbaciones del asma cursan con periodos de una baja ingesta de líquidos y de vómitos con deshidratación asociada. La deshidratación incrementa la acidosis metabólica, por lo que se debe de corregir el estado hídrico del paciente hacia una normovolemia con el uso de líquidos orales (preferentemente) o por vía intravenosa. Se debe de tener en cuenta que una sobrehidratación incrementa el riesgo de provocar edema pulmonar, además de que en las crisis asmáticas severas se puede presentar un síndrome de secreción inapropiada de la hormona antidiurética, por lo que se debe de mantener un balance hidroelectrolítico ${ }^{22}$.

La terapia broncodilatadora es la parte más importante y la primera línea de tratamiento farmacológico en el tratamiento inicial con crisis asmática severa ${ }^{23}$. El salbutamol es un beta-2 agonista y es el agente broncodilatador que se usa con mayor frecuencia $^{21}$. Se usa en forma de micronebulizaciones $2.5 \mathrm{mg}$ en solución salina al $9 \%$. La vía de administración es preferentemente con tienda facial. En la terapia inicial la frecuencia de las microneublizacines estará determinada por la respuesta observada en 1-2 $\mathrm{hrs}^{1}$. Se ha documentado que las terapias inhalatorias continuas con beta- 2 agonistas son más eficaces y con efecto broncodilatador más prolongado que la terapia intermitente $^{28}$. La dosis recomendada en niños para la terapia inhalatoria continua es de $0.15 \mathrm{mg} / \mathrm{kg}$ en $5 \mathrm{ml}$ de solución fisiológica al $9 \%{ }^{25}$, además de que se recomienda iniciarla durante la primera o las primeras horas del tratamiento y después continuar con inhalaciones intermitentes a demanda ${ }^{1}$. Los efectos adversos con el uso de beta- 2 agonistas en el asma se relacionan con el sistema cardiovascular, e incluyen taquicardia, incremento del intervalo QT en el electrocardiograma, arritmias, hipertensión o hipotensión arterial; además de presentar efectos sobre el sistema nervioso (ansiedad, temblor, náusea y vómito), en el equilibrio electrolítico y metabólico (hipocalemia, hiperglucemia) ${ }^{22,23,28}$.

Los fármacos anticolinérgicos bloquean la bronconstricción y la secreción de moco mediadas por la acción del sistema parasimpático en el árbol bronquial. El bromuro de ipratropio es el anticolinérgico más usado en las exacerbaciones de asma y se puede usar en nebulización en combinación con un beta-2 agonista, ya que ha mostrado ser mejor que el tratamiento en monoterapia ${ }^{29}$ Se usan 2 ó 3 dosis de 250 a 500 microgramos en $2-5 \mathrm{ml}$ de solución salina $0.9 \%$ administrados cada 20 minutos durante una hora y posteriormente cada cuatro ho- 
$\operatorname{ras}^{1,21-23}$. El uso de bromuro de ipratropio se debe de considerar cuando los síntomas son refractarios al uso de beta-2 agonistas o en exacerbaciones moderadas-severas ${ }^{1,22}$. Los efectos secundarios más frecuentes son boca seca, sabor amargo, rubor, taquicardia y discinesias ${ }^{24}$.

Los esteroides sistémicos son la primera línea de tratamiento en las exacerbaciones asmáticas severas junto con los agonistas de los receptores beta adrenérgicos ${ }^{23}$. Aceleran la resolución de la exacerbación, previenen la recaída y disminuyen la mortalidad $^{21}$. Inhiben la inflamación a nivel celular y disminuyen la hiperreactividad de las vías aéreas, normalizan la proporción entre células ciliadas-células secretoras de moco e incrementan los efectos de los beta-2 agonistas, ya que aumenta la expresión de los receptores beta- 2 en el músculo liso bronquial y previene la regulación a la baja de los mismos por el uso prolongado de beta agonistas ${ }^{21,23}$. Se puede usar prednisona 1-2 $\mathrm{mg} / \mathrm{kg} /$ día (máximo 20 mg en niños menores de 2 años; 30 mg en niños de 2-5 años y $40 \mathrm{mg}$ en niños mayores de 5 años de edad) vía oral por 3-5 días ${ }^{22}$, en caso de intolerancia a la vía oral, metilprednisolona a dosis de $1 \mathrm{mg} / \mathrm{kg}$ cada 6 horas en el primer día ${ }^{1,29} y$, en caso de control del cuadro agudo, continuar con prednisona vía ora|21,23. Se recomienda no usar corticoesteroides por más de 5 días, ya que a corto plazo las dosis altas pueden producir hiperglucemia, hipertensión arterial y psicosis aguda, y el uso prolongado de los mismos puede producir inmunosupresión, supresión del eje hipotálamo-hipófisis-adrenal, osteoporosis, miopatía y debilidad ${ }^{23,24,29}$. No existe suficiente evidencia sobre la eficacia de los esteroides inhalados sobre la terapia sistémica, pero cuando se utilizan en etapas tempranas del cuadro a grandes dosis pueden reducir la tasa de admisiones hospitalarias en pacientes con crisis asmática moderada-severa ${ }^{23}$, y se utiliza budesonida $200 \mu \mathrm{cg}$ por dosis ${ }^{21}$.

El uso de sulfato de magnesio tiene un potencial beneficio durante las agudizacio- nes del asma, ya que produce relajación del músculo liso bronquial secundario a la inhibición de la entrada de calcio al mismo ${ }^{22}$, sin embargo, no se recomienda su uso rutinario en niños menores de 5 años con exacerbaciones asmáticas ${ }^{29}$. Se utiliza en pacientes con crisis asmática severa con respuesta incompleta o sin respuesta al tratamiento inicial durante las primeras 2 horas $^{22,23}$. La dosis utilizada es de $40-50 \mathrm{mg} / \mathrm{kg}$ (máximo 2 g) para pasar en 20-60 minutos ${ }^{1}$. La hipotensión secundaria al uso de magnesio es infrecuente. En una reciente revisión Cochrane se concluye que las nebulizaciones de sulfato de magnesio con un beta- 2 agonista, mejoran la función pulmonar y disminuyen la tasa de hospitalizaciones en pacientes con asma severa y se considera su uso como tratamiento adyuvante estándar en niños de 2 años o más de edad con exacerbaciones severas, particularmente aquellos con sintomatología $<6 \mathrm{hrs}^{1,30}$. Los niveles séricos de magnesio se deben de monitorear de forma regular si se consideró el tratamiento intravenoso en infusión continua, ya que con concentraciones elevadas (>10-12 mg/dl) pueden aparecer complicaciones severas, como arritmias cardiacas, falla respiratoria por debilidad muscular o paro cardiorrespiratorio súbito ${ }^{24}$.

La adición de helio al oxígeno (heliox, mezcla de $70 \%$ helio y $30 \%$ oxígeno) disminuye la densidad de los gases inspirados e incrementa de forma importante el flujo laminar, y teóricamente esto ayuda a disminuir el trabajo respiratorio y a mejorar la eficacia de la terapia inhalatoria ${ }^{23,29}$. A pesar de que los estudios realizados sobre el uso de heliox en las exacerbaciones asmáticas han mostrado resultados contradictorios, se considera su uso en niños mayores de 5 años en quienes las exacerbaciones amenazan su vida o en exacerbaciones severas que no remiten tras una hora de tratamiento intensivo con la terapia convencional; sin embargo, su uso se encuentra en debate ${ }^{1,31}$.

En las guías actuales Global Strategy for Asthma Management and Prevention 
(GINA 2015) las metilxantinas no se consideran como parte del manejo de las crisis asmáticas en niños; esto debido a que se tienen que monitorear los niveles séricos del medicamento (rangos terapéuticos-tóxicos estrechos), alta incidencia de toxicidad, el incremento de tiempo de hospitalización y los numerosos efectos adversos ${ }^{23}$.

En pacientes en los que no responden o lo hacen de forma parcial al tratamiento de urgencia estándar se pueden añadir antagonistas del receptor de leucotrieno, como montelukast o zafirlukast ${ }^{25,26}$. A pesar de que este tipo de medicamentos se usa convencionalmente para el tratamiento crónico del asma, existen publicaciones que recomiendan su uso en las exacerbaciones agudas ya que, a pesar de no haber diferencias clínicas significativas, se ha observado que mejora la función pulmonar y sus efectos adversos son mínimos $28,32,34$. Sin embargo, se requiere de mayor evidencia para recomendar su uso.

En niños mayores de 5 años con dificultad respiratoria severa, el uso de BPAP (Bilevel Positive Airway Pressure) puede facilitar la administración de beta-2 agonistas inhalados, sobre todo en áreas pobremente ventiladas. El uso de BPAP es seguro y generalmente bien tolerado, puede mejorar la oxigenación y disminuir el trabajo respiratorio, por lo que su uso de forma oportuna puede ser una alternativa de la ventilación mecánica invasiva ${ }^{23}$. Los parámetros recomendados incluyen una presión positiva en inspiración (IPAP, del inglés Inspiratory Positive Airway Pressure) de $10 \mathrm{cmH}^{2} \mathrm{O}$, presión positiva en expiración (EPAP, del inglés Expiratory Positive Arway Pressure) de 5 $\mathrm{CmH}_{2} \mathrm{O}$ y uso de presión positiva continua en la vía aérea (CPAP, del inglés Continuos Positive Arway Pressure) para disminuir el cierre prematuro de la vía aérea, disminuir la presión intrínseca al final de la espiración y el trabajo respiratorio ${ }^{8}$

La ketamina es un antagonista no competitivo de los receptores ionotrópicos de glutamato (NMDAr) usado como anesté- sico, analgésico y sedante con un amplio índice terapéutico y efectos hemodinámicos favorables ${ }^{8}$. Posee efecto de broncodilatación y de aclaramiento mucoso. La ketamina es una excelente opción en pacientes que requieren intubación con secuencia rápida gracias a que disminuye la respuesta broncoconstrictora por la inserción del tubo endotraqueal ${ }^{21}$. La dosis aún no está estandarizada, pero en una revisión reciente se recomienda la administración intravenosa de $0.1-2 \mathrm{mg} / \mathrm{kg}$ en bolo seguido de infusión continua de $0.15-2.5 \mathrm{mg} / \mathrm{kg} / \mathrm{hr}^{35}$. Los efectos secundarios incluyen hipoventilación, hipertensión, laringoespasmo, agitación, incrementa el flujo sanguíneo cerebral con incremento de la presión intracraneal e intraocular consecuentemente ${ }^{23}$.

La intubación y ventilación mecánica asistida es necesaria en niños con síntomas refractarios a la terapia convencional y paro respiratorio inminente ${ }^{23}$. Las indicaciones para intubación en niños con crisis asmática severa son: 1) pobre respuesta a la terapia convencional; 2) disminución o ausencia de ruidos respiratorios; 3) hipercapnia severa $\left(\mathrm{PCO}_{2}>50 \mathrm{mmHg}\right) ; 4$ ) hipoxia severa $\left.\left(\mathrm{PO}_{2}<60 \mathrm{mmHg}\right) ; 5\right)$ deterioro del estado mental; 6) fatiga de los músculos respiratorios; 7) paro respiratorio inminente o apnea; y 8) paro cardiorespiratorio ${ }^{23,24}$. Los puntos a considerar previa intubación son la preoxigenación manteniendo una saturación al $100 \%$, accesos intravenosos disponibles, considerar la administración de bolos de solución intravenosa para prevenir la hipotensión aguda secundaria a la intubación por reflejo vagal y escoger el tamaño del tubo endotraqueal adecuado (diámetro interno $=[$ edad en años/4] +3.5 ). Se debe de tener en cuenta medicación previa a la secuencia rápida de intubación, ya que ayuda a atenuar los efectos adversos de la laringoscopia y la intubación. La administración de atropina en niños menores de 8 años puede prevenir la bradicardia secundaria a la intubación endotraqueal; así como el uso de lidocaína en cualquier edad disminu- 
ye el broncoespasmo reflejo ${ }^{26}$. La ketamina es el medicamento sedante preferido como agente inductor en pacientes con asma severa por sus efectos broncodilatadores; mientras que el rocuronio es el bloqueador neuromuscular que se prefiere, ya que po- see pocos efectos secundarios y por su inicio de acción rápida y prolongada, pero el vecuronio es otro bloqueador neuromuscular que se usa comúnmente y puede proveer de una adecuada parálisisis,24.

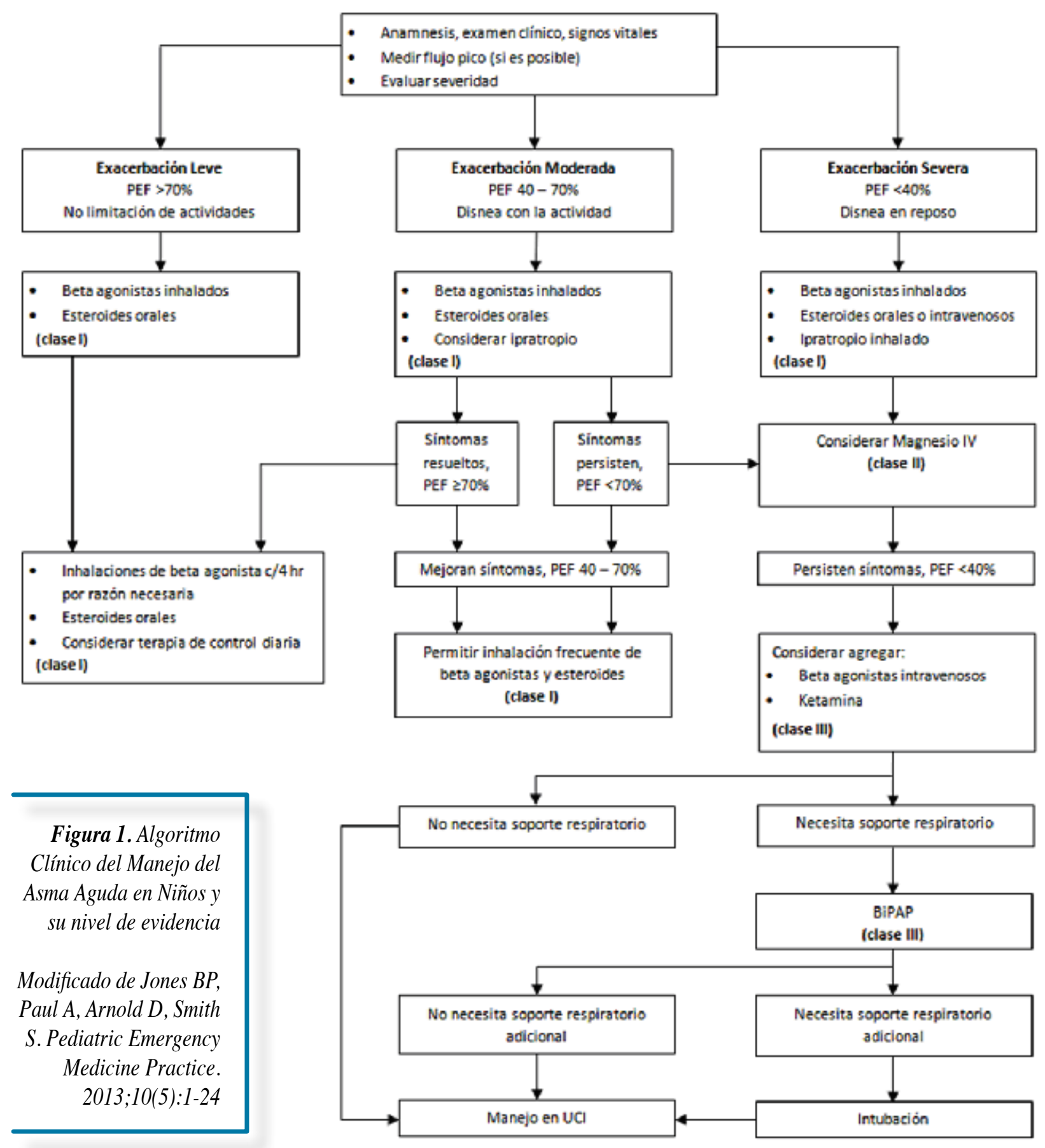


El objetivo de la ventilación mecánica es prevenir la fatiga progresiva de los músculos respiratorios, reducir el consumo de oxígeno, incrementar el volumen pulmonar, incrementar el $\mathrm{CO}_{2}$ exhalado y el $\mathrm{O}_{2}$ inhalado, mejorar la función cardiopulmonar, y limpiar las secreciones de la vía aérea ${ }^{21}$. Los ajustes recomendados del ventilador mecánico son los siguientes: 1) modo volumen control SIMV (del inglés Syncronized Intermittent Mechanical Ventilation), ayuda a prevenir el barotrauma; 2) volumen corriente $5-6 \mathrm{~mL} / \mathrm{kg}$; 3) frecuencia respiratoria igual a la media normal esperada para la edad; 4) índice inspiración:espiración $1: 3$; 5) PEEP $0-3 \mathrm{~cm} \mathrm{H}_{2} \mathrm{O}^{23,24}$. Las complicaciones más frecuentes de ventilación mecánica en los niños con estado asmático son hipotensión, desaturación de oxígeno, neumotórax, enfisema subcutáneo y paro cardiaco. Si el paciente presenta hipotensión y/o hipoxemia que no responden a la administración de fluidos o al cambio de los parámetros ventilatorios, se deberá de considerar un neumotórax a tensión ${ }^{23}$. Las metas a considerar en un paciente asmático intubado son mantener una adecuada oxigenación con saturación de oxígeno mayor al $91 \%$, mantener un $\mathrm{pH}$ de sangre arterial de $>7.2$ y promover el uso de hipercapnia permisiva ${ }^{23,24}$.

El omalizumab es un anticuerpo monoclonal humanizado de tipo IgG que se une con gran afinidad a los receptores de IgE en la superficie de los mastocitos y basófilos $^{36}$. Las guías sobre el tratamiento del asma sólo recomiendan su uso en pacientes mayores de 12 años de edad con asma severa persistente mediada por IgE ${ }^{37}$ pero no se cuentan con estudios de alto impacto sobre su uso en exacerbaciones agudas del asma.

El éxito relativo que ha tenido el tratamiento con agentes biológicos como el omalizumab ha generado un gran interés en el potencial terapéutico que tiene este tipo de fármacos en el manejo del asma.
Se han desarrollado numerosos agentes que actualmente se encuentran en estudio como los anticuerpos tipo IgG contra IL-5, IL-13, IL-4, IL-9, IL-17 y contra el TNF- $\alpha^{38}$. Hasta el momento el dupilumab, un anticuerpo monoclonal tipo IgG contra el receptor de IL-4, ha mostrado efectos positivos en el tratamiento de pacientes con asma moderada-severa persistente, disminuyendo las exacerbaciones agudas, la sintomatología y los biomarcadores de inflamación de tipo $\mathrm{TH}_{2}$, y mejorando la función pulmonar ${ }^{28}$.

\section{Complicaciones}

La complicación aguda más común es la atelectasia secundaria a taponamiento mucoso. Los pacientes que han requerido de ventilación asistida con presión positiva en la vía aérea pueden presentar fugas de aire, como el neumomediastino y/o neumotórax, que pueden poner en riesgo la vida de los pacientes. El neumotórax se puede sospechar en pacientes con deterioro súbito asociado a dolor torácico, asimetría de los ruidos respiratorios o retracción traqueal ${ }^{12}$. También se pueden presentar complicaciones cardiacas no asociadas a efectos adversos de los fármacos utilizados para el tratamiento de las exacerbaciones agudas del asma, como arritmias y paro cardiaco $^{20,26}$. Las complicaciones secundarias al uso de fármacos para el tratamiento ya se han descrito en párrafos anteriores.

\section{Pronóstico}

La mortalidad por crisis asmática va desde 0-22\%, con menor mortalidad documentada en aquellos pacientes en los que se empleó hipercapnia permisiva. La mortalidad se asocia positivamente en los pacientes que presentan $\mathrm{pH}$ inicial bajo y $\mathrm{PaCO}_{2}$ alta al ingreso hospitalario, y con el desarrollo de falla multiorgánica ${ }^{12}$. La duración de la estancia hospitalaria varía, reportándose que la mayor duración se asocia con el género femenino, pacientes que requi- 
rieron intubación y apoyo ventilatorio, la administración de bloqueadores neuromusculares por más de 24 horas y el uso de corticoides inhalados previo a su ingreso a la UCIP12,14.

\section{Conclusiones}

A pesar de que el asma es uno de los principales padecimientos pulmonares y sus exacerbaciones agudas, una de las causas más frecuentes de hospitalización en la edad pediátrica, las guías sobre el manejo de las crisis asmáticas se basan en el tratamiento sintomático de las mismas. Actualmente, se comprende mejor la imunobiología y la fisiopatología del asma y se han definido los genotipos y fenotipos individuales en los pacientes con asma. Por ello, la heterogeneidad de la respuesta de los pacientes al manejo convencional sugiere que, como en cualquier área de la medicina, se tenga que individualizar el tratamiento. Son necesarios más ensayos clínicos con agentes biológicos, tanto en el manejo crónico del asma como en sus exacerbaciones para poder ofrecer un tratamiento más curativo y menos paliativo. De esta forma se podrá mejorar la calidad de vida de los pacientes y sus familias y disminuir la gran carga económica que representa esta enfermedad para los sistemas de salud.

\section{Bibliografía}

1. Global Strategy for Asthma Management and Prevention in Children 5 Years and Younger. GINA 2015.

2. Bigham M, Brili R. Status asthmaticus En: Roger's Textbook of Pediatric Intensive Care. Lippincott Williams \& Wilkins, Philadelphia PA, USA,2008, pág. 686-696.

3. Kenyon N. Zeki AA. Albertson TE. Louie S. Definition of Critical Asthma Syndromes. Clinic Rev Allerg Immunol. 2015;48:1-6.

4. del Río-Navarro BE. Hidalgo-Castro EM. SienraMonge JJL. Asma. Bol Med Hosp Infant Mex [revista en la Internet]. 2009 Feb [citado 2015 Oct 10] ; 66( 1 ): 3-33. Disponible en: http://www.scielo. org. $\mathrm{mx} /$ scielo.php?script $=$ sci_arttext $\&$ pid $=\mathrm{S} 1665$ $11462009000100002 \& \operatorname{lng}=$ es.

5. del Río-Navarro BE. del Río-Chivardi JM. Berber A. Sienra-Monge JJ. Rosas-Vargas MA. Baeza-Bacab $M$. Asthma prevalence in children living in north Mexico City and a comparison with other Latin American cities and world regions. Allergy Asthma Proc.2006;27(4):334-40.

6. Owens PL. Thompson J. Elixhauser A. Ryan K. Care of Children and Adolescents in U.S. Hospitals. Report No. AHRQ Publication No. 04-0004. Rockville, MD: AHRQ; 2003.
7. Bratton SL. Odetola FO. McCollegan J. Cabana MD. Levy FH. Keenan HT. Regional variation in ICU care for pediatric patients with asthma. J Pediatr 2005; 147:355-361

8. Fernández Nievas IF. Anand K. Severe Acute Asthma Exacerbation in Children: A Stepwise Approach for Escalating Therapy in a Pediatric Intensive Care Unite. J Pediatr Pharmacol Ther 2013;18(2):88-104.

9. Harper RW. Zeki AA. Immunobiology of the Critical Asthma Syndrome. Clinic Rev Allerg Immunol. 2015;48:54-65.

10. Galowitz S. Chang C. Immunobiology of Critical Pediatric Asthma. Clinic Rev Allerg Immunol. 2015;48:84-96.

11. Murphy DM. O'Byrne P. Recent Advances in the Pathophysiology of Asthma. CHEST 2010; 137(6):1417-1426.

12. Sánchez Eng L. Asma grave. En: Temas Selectos en Terapia Intensiva Pediátrica, Tomo I. Asociación Mexicana de Terapia Intensiva Pediátrica. $1^{\text {a }}$ Edición. Editorial Alfil. México, D.F., 2013, pág. 255-264.

13. Avdalovic M. Pulmonary Vasculature and Critical Asthma Syndromes: a Comprehensive Review. Clinic Rev Aller Immunol. 2015:48:97-103.

14. Stevenson MD, Ruddy RM. Asthma and Allergic Emergencies. En Fleishner GR, Ludwing S: Textbook of Pediatric Emergency Medicine. Lippincott Williams \& Wilkins. Philadelphia, USA 2010. Pp 649-659. 
15. Moser S. Peroni D. Comberiati P. Piacentini G. Asthma and viruses: is there a relationship?. Frontiers in Bioscience 2014;6:46-54.

16. Sandrock CE. Norris A. Infection in Severe Asthma Exacerbations and Critical Asthma Syndrome. Clinic Rev Allerg Immunol. 2015;48:104-113.

17. Kurai D. Saraya T. Ishii H. Takizawa H. Virus-induced exacerbations in asthma and COPD. Front.Microbiol. 2013;4(293):1-12.

18. Shah KK. Dishop MK. Infantile Viral Illnesses. En A.E. Fraire et al. (eds.), Viruses and the Lung, SpringerVerlag Berlin Heidelberg 2014, pág. 143-158.

19. Prescott Atkinson T. Is Asthma an Infectious Disease? New Evidence. Curr Allergy Asthma. 2013; 13:702-709.

20. Adams JY. Sutter ME. Albertson TE. The Patient with Asthma in the Emergency Departament. Clinic Rev Allerg Immunol. 2012;43:14-29.

21. Wang XF. Hong JG. Management of severe asthma exacerbation in children. World J Pediatr 2011;7(4):293-301.

22. Øymar K. Halvorsen T. Emergency presentation and management of acute severe asthma in children. Scandinavian Journal of Trauma, Resuscitation and Emergency Medicine. 2009;17(40):1-11.

23. Jones BP. Paul A. Arnold D. Smith S. Management Of Acute Asthma In The Pediatric Patient: An Evidence-Based Review. Pediatric Emergency Medicine Practice. 2013;10(5):1-24.

24. Saharan S. Lodha R. Kabra SK. Management of Status Asthmaticus in Children. Indian J Pediatr. 2010;77:1417-1423.

25. Albertson TE. Sutter ME. Chan AL. The Acute Management of Asthma. Clinical Rev Allerg Immunol. 2015;48:114-125.

26. Schivo M. Phan C. Louie S. Harper RW. Critical Asthma Syndrome in the ICU. Clinic Rev Allerg Immunol. 2015;48:31-44.
27. Corrales AY. Soto-Martínez M. Starr M. Management of severe asthma in children. Australian Family Physician.2011;40(1/2):35-38.

28. Albertson TE. Schivo M. Gidwani N. et al. Pharmacotherapy of Critical Asthma Syndrome: Current and Emerging Therapies. Clinic Rev Allerg Immunol. 2015;48:7-30.

29. Wade A. Chang C. Evaluation and Treatment of Critical Asthma Syndrome in Children. Clinic Rev Allerg Immunol. 2015;48:66-83.

30. Powell C. Dwan K. Milan SJ. et al. Inhaled magnesium sulfate in the treatment of acute asthma. Cochrane Database Syst Rev. 2012: CD003898.

31. National Asthma Education and Prevention Program (2007) Expert Panel Report 3 (EPR-3): Guidelines for the diagnosis and management of asthma-summary report 2007. J Allergy Clin Immunol 120:S94138.

32. Wheeler DS. Lutfi R. Status Asthmaticus. En: Pediatric Critical Care Medicine Vol. 2: Respiratory, Cardiovascular and Central Nervous Systems. SpringerVerlag London 2014, pág. 49-73.

33. Camargo CA Jr. Gumer DM. Smithline HA. et al. A randomized placebo-controlled study of intravenous montelukast for the treatment of acute asthma. Allergy Clinic Immunol. 2010;125(2):374-380.

34. Ramsay CF. Pearson D. Mildenhall S. Wilson AM. Oral montelukast in acute asthma exacerbations: a randomized, doubleblind, placebo-controlled trial. Thorax. 2010;66(1):7-11.

35. Goyal S. Agrawal A. Ketamine in status asthmaticus: A review. Indian J Crit Care Med 2013;17:154-61.

36. Holgate ST. Polosa R. Treatment strategies for allergy and asthma. Nat Rev Immunol. 2008;8:218-230.

37. Díaz R. Charles Z. George E. Adler A. NICE guidance on omalizumab for severe asthma. Lancet Respir Med. 2013;1:189-190.

38. Pelaia G. Vatrella A. Maselli R. The potential of biologics for treatment of asthma. Nat Rev Drug Discov. 2012;11:958-972. 